Professor Emeritus

University of Agriculture

Faisalabad.

\author{
Correspondence Address: \\ Bakht Baidar Khan \\ Professor Emeritus \\ University of Agriculture \\ Faisalabad. \\ Article received on: \\ 01/06/2016 \\ Accepted for publication: \\ 30/06/2016 \\ Received after proof reading: \\ 04/07/2016
}

\section{WHERE PATIENTS MATTER}

\begin{abstract}
Bakht Baidar Khan
\end{abstract}
Article Citation: Khan BB. Where patients matter. Professional Med J 2016;23(7):000-000.

Article Citation: Khan BB. Where patients matter. Professional Med J 2016;23(7):763-765. DOI: 10.17957/TPMJ/16.3482
The observations presented herein are the direct outcome of author's personal experience gained during admission in five different hospitals in the US and Canada on different occasions during the last three years. Admission in a hospital for treatment of various ailments is usually done through Emergency Room (ER). For gaining entrance to ER, some kind of health insurance is required for each individual. However, in an emergent situation, even an illegal immigrant may be entertained for health care. Those on duty in ER will be found ever ready to receive, identify, register and guide all incoming patients very politely without any greed or grudge, during day and night, of course, under shift arrangements. Before entrance to $\mathrm{ER}$, an identification tag made of plastic with patient's name and date of birth on it, is placed around his wrist. This tag must stay in place during patient's stay in the hospital. The golden principle of making a queue is strictly observed. Never a person would think of going ahead without his/her turn. Very unstable and really aged people may be dealt with separately.

Preliminary diagnostic investigations are carried out by final year Residency students in consultation with senior doctors. Blood analysis, X-rays and some orthopedic help are immediately available. If need be, patients are referred to for sonography, C.T. Scan, etc. Such cases that need further regular medical care, they are admitted and sent to the wards concerned, others exhibiting reasonable improvement are discharged from ER and sent home.
Heart problem cases, fractures, semi-and fully unconscious patients are attended on priority with no distinction of any type relative to colour, creed, or caste. Getting medical aid at a public hospital is considered ones birth right.

When the patient is in ER or admitted in hospital, no nurse or doctor will ask a patient to bring a branula, an injection, or any medicine from the market. Quality medicines, injections, etc. are always available in hospital pharmacy. On the other hand, no indoor patient is allowed to use any medicine on his own other than that prescribed by the doctor concerned.

During the course of treatment, patients may be referred to other departments for various tests, procedures, or use of diagnostic tools such as CT scan, MRI. The patients are taken to such departments either in a wheel chair or on a bed secured well from sides and partly covered with a clean sheet. Members of transport team perform this duty absolutely without any expectation of tip or Bakhshish, a word completely culled out from hospital terminology in USA/Canada.

Outdoor patients are handled in outdoor clinics such as blood clinic, medical clinic, and surgery clinic. Patients without any insurance have to pay registration fee. Then get a token from the concerned clinic and wait for their turn to see the doctor. No recommendation or phones from high ups are required to visit or consult a doctor.

Doctors, nurses and other junior staff including 
janitors are so dutiful that their punctuality and polite behavior make the patients feel assured to get well soon unless luck has deserted someone. Normally, two patients are kept in a room, which includes toilet and shower as well. The rooms are cleaned twice daily. While under treatment in the hospital, the author had to stay for eleven days in a room, which was also shared by another elderly patient. He was not a stable patient. The nurse on duty and her juniors had to change the bedding and his diapers (underpants) twice a day. They were doing this with a high sense of devotion. Also, they had to feed the patient the food provided by the hospital as ordered by his doctor. During the eleven days when I was with him, not a single relative or friend visited him, but he seemed content, simply because he was getting the best hospital care and medication.

In October, 2014, I was again admitted in a hospital for blood in stools. I personally witnessed an incidence, which was vividly different from the one just mentioned above. I was allotted a bed in a room where already another patient was present. It was already $01 \mathrm{AM}$ and the other patient was asleep. In the morning we exchanged hello/hi with each other. He seemed to have a woeful appearance.

At about midnight, a nurse assistant came to record his blood pressure, but he became furious and blamed the nurse for stealing his hundred dollar bill. He made a lot of fuss with loud noise. Nurses and other workers gathered there and cooled him. Next morning, the nurse on duty went to him to administer his medication, but for nothing the patient became very impatient and started wrestling with her. The nurse was young and strong, she put him flat on the floor. In the meantime, a crowd of nurses, doctors and other workers had gathered there. Also, police reached the scene. Finally, the furious patient begged for mercy and was excused.

The greatness of the system lies in the fact that neither the discourteous patient was thrown out of the hospital nor was his treatment discontinued. I was told that according to law, he cannot be denied treatment in the hospital. The author feels that the above incidence should be an "eyeopener" in societies like ours.

Cleanliness is order of the day. Bedding of each patient is usually changed after two days, while setting of bedding is done once a day. Gloves have to be put on by nurses before touching the patient for any purpose such as injecting him a medicine, taking his blood sample, or bandaging him. The nurse has to reaffirm each time the identity of the patient by looking at the identity tag around his wrist, so that the medicine is administered to the right patient. Patients suspected of having some infectious problem are kept as a single patient in a room. All types of staff including doctors need to put on a yellowcoloured overall and a mask while visiting such a patient. Additional precautionary measures may also be adopted depending on the situation.

The duty of nurses changes every morning and evening. When the next nurse takes change of her patients, the outgoing reviews with the next nurse all important information about each patient in her charge. Nurses play a vital role in overall patient care. Any laxity on the part of a nurse can cost loss of her nursing license. Nurses have to be there before the doctors start visiting the patients. Before the visit of a senior doctor, senior residency students or PAs pay a visit to each patient in their respective wards. Then a little later, senior doctors along with residency students discuss with each patient about the progress made or about the required changes in medication. Any laxity on the part of a doctor is taken very seriously. A committee probes the matter and a proven slackness subjects the doctor to face various penalties under the hospital rules. Dissatisfied patients can knock the door of a court against the doctor.

Except such medicines that are available at the counter, all others need a prescription by a qualified doctor (MD), possessing a license. Having simply the degree of MBBS, does not qualify a person to practice medicine or prescribe drugs in USA or Canada. It would not be out of 
place to mention have that Quackery has no room in these countries, where at every step it is reminded that "it is Law to do this", "It is Law to do that ". Quackery is a curse, it finds its way only in societies that are not law abiding and where people are ignorant of its damaging effects.

Only qualified people can run a pharmacy shop. Except such medicines that are available at the counter, no other medicine can be obtained from a pharmacy shop without 'doctor's prescription.

The food service of a hospital provides breakfast, lunch and dinner to all needy indoor patients unless a patient himself refuses to eat hospital food because of certain restrictions. The nurse or doctor in question has to provide the names of patients to food service so as to arrange food for them. Those desirous of eating Halal food may clearly indicate to the nurse concerned and she would manage it for them.

No visitors are allowed to see their patients before 11 AM each day. However, the rest of day and up to $9 \mathrm{PM}$, one person, turn by turn, can visit the patient. Permission to visit a patient is by pass.

A well-managed ambulance service exists, which is run both by the hospital as well as private parties. Just give a phone call and the ambulance is at your door step. City police is always helpful, especially for patients in precarious condition. Police help is available at phone \# 911.

Aged patients having no body to take care of them at home or chronic patients requiring treatment for extended periods or old patients who are otherwise well-off but the dear ones are too busy to look after them, may be housed in Nursing Homes. One or two nurses depending on the number of patients, are always on duty. Doctors pay regular visits to nursing homes, some as volunteers while others as a part of their duty. Food is served to patients according to the advice of doctors. Some patients or residents get food from their homes.

Service charges for chronic unstable patients are met through some insurance schemes, while those who reside in a nursing home of their own choice, they are charged for all services provided to them.

The information provided in this article is the outcome a personal observations.

\section{AUTHORSHIP AND CONTRIBUTION DECLARATION}

\begin{tabular}{c|l|c|c|}
\hline Sr. \# & \multicolumn{1}{|c|}{ Author-s Full Name } & Contribution to the paper & Author=s Signature \\
\hline 1 & Bakht Baidar Khan &
\end{tabular}

